\title{
ON A FAMILY OF SPECIAL LINEAR SYSTEMS ON ALGEBRAIC CURVES ${ }^{1}$
}

\author{
EDMOND E. GRIFFIN II
}

\begin{abstract}
L \rightarrow C$ of smooth algebraic curves $C$ of genus 10 together with line bundles $L$ of degree 6 such that $H^{(1)}(C, L) \geqslant 3$. It is shown that one of the irreducible components of this scheme is nonreduced at every point.
\end{abstract}

Introduction. In [ACGH] a general method is outlined for computing the dimension of the tangent space $T_{l}\left(\psi \psi_{d, g}^{r}\right)$. $\partial \int_{d, g}^{r}$ is the scheme parametrizing pairs $L \rightarrow C$ in which $C$ is a smooth curve of genus $g, L$ is a line bundle such that

(1) $\operatorname{deg}_{C}(L)=d$,

(2) $\operatorname{dim} H^{0}(C, L)=h^{0}(C, L) \geqslant r+1$,

and $l=L \rightarrow C$ is a "generic" point on $\mathcal{Q} \int_{d, g}^{r}$. Specifically, $l \in \mathcal{Q} \int_{d, g}^{r}-\mathcal{U} \int_{d, g}^{r+1}$.

The dimension of this tangent space is determined by the kernels of two naturally defined maps on the cohomology of $C$ in $L$ and $K_{C}$. These maps, $\mu_{0}$ and $\mu_{1}$, together with their kernels will be computed explicitly in the case $l \in \mathcal{Q} \int_{6,10}^{2}-\mathcal{Q} \int_{6,10}^{3}$ and $C$ a hyper-elliptic curve. This calculation will then be used to show that $2 \int_{6,10}^{2}$ has a nonreduced component.

It will be clear that these computations generalize to higher genus and degree hyper-elliptic curves, but the actual calculations become fairly intractable. For this reason only the case of $2 \int_{6,10}^{2}$ will be considered here.

1. We begin with a review of the basic set up and terminology of [ACGH]. For a fixed, smooth curve $C$ of genus $g$, define

$$
\begin{aligned}
& \operatorname{Pic}^{d}(C)=\left\{L \mid \operatorname{deg}_{C}(L)=d\right\} \\
& \cup \mid \\
& W_{d}^{r}(C)=\left\{L \mid \operatorname{deg}_{C}(L)=d \text { and } h^{0}(C, L) \geqslant r+1\right\} .
\end{aligned}
$$

It is well known that

$$
T_{L}\left(\operatorname{Pic}^{d}(C)\right) \simeq H^{1}\left(C, \theta_{C}\right)
$$

Received by the editors April 25, 1983.

1980 Mathematics Subject Classification. Primary 14H15, 14D15, 14H45.

'This work forms part of the author's doctoral thesis, written at Harvard University in 1982 under the advice of Phillip Griffiths. 
A first order variation of a line bundle $L$ on $C$ is a commuting diagram,

$$
\begin{array}{ccc}
L & \rightarrow & \varrho \\
\downarrow & & \downarrow \\
C & \rightarrow & C \times S_{1} \\
\downarrow & & \downarrow \\
S_{0} & \rightarrow & S_{1}
\end{array}
$$

where $S_{n}=\operatorname{Spec}\left(\mathbf{C}[t] /\left(t^{n+1}\right)\right)$. The set of isomorphism classes of such diagrams can be canonically identified with $H^{1}\left(C, \theta_{C}\right)$.

The obstruction to extending a section $s \in H^{0}(C, L)$ to a section of $E$ can be seen to be $\varphi \cdot s \in H^{1}(C, L)$, where

$$
\text { “. } H^{1}\left(C, \vartheta_{C}\right) \otimes H^{0}(C, L) \rightarrow H^{1}(C, L)
$$

is the usual cup product, and $\varphi \in H^{\mathrm{l}}\left(C, \Theta_{C}\right)$ is the element corresponding to $[$. Thus, one sees that, at a point $L \in W_{d}^{r}(C)-W_{d}^{r+1}(C)$, a tangent vector $\varphi$ in $H^{1}\left(C, \Theta_{C}\right)=T_{L}\left(\mathrm{Pic}^{d}(C)\right)$ is also in $T_{L}\left(W_{d}^{r}(C)\right)$ if and only if $\varphi: H^{0}(C, L) \rightarrow$ $H^{1}(C, L)$ is the zero map. In other words, $\varphi$ is in the kernel of the map

$$
H^{1}\left(C, \vartheta_{C}\right) \rightarrow \operatorname{Hom}\left(H^{0}(C, L), H^{1}(C, L)\right) .
$$

Dualizing yields the map

$$
\mu_{0}: H^{0}(C, L) \otimes H^{0}\left(C, K L^{-1}\right) \rightarrow H^{0}(C, K)
$$

such that

$$
T_{L}\left(W_{d}^{r}(C)\right)=\left(\text { Image } \mu_{0}\right)^{\perp}
$$

in $h^{0}(C, L)=r+1$.

Now the Riemann-Roch theorem implies $h^{0}\left(C, K L^{-1}\right)=g-d+r$. So,

$$
\begin{aligned}
\operatorname{dim}\left(\operatorname{Im} \mu_{0}\right)^{\perp} & =g-\operatorname{dim}\left(\operatorname{Im} \mu_{0}\right)=g-\left((r+1)(g-d+r)-\operatorname{dim}\left(\operatorname{ker} \mu_{0}\right)\right) \\
& =(g-(r+1)(g-d+r))+\operatorname{dim}\left(\operatorname{ker} \mu_{0}\right)=_{\operatorname{def}} \rho+\operatorname{dim}\left(\operatorname{ker} \mu_{0}\right) .
\end{aligned}
$$

The number $\rho$ is called the Brill-Noether number. So, at a generic $L$,

$$
\operatorname{dim} T_{L}\left(W_{d}^{r}(C)\right)=\rho+\operatorname{dim}\left(\operatorname{ker} \mu_{0}\right) .
$$

Next we allow $C$ to vary and define (with $g$ fixed)

$$
\begin{aligned}
& \operatorname{Pic}^{d}=\left\{L \rightarrow C \mid \operatorname{deg}_{C}(L)=d, g(C)=g\right\} \\
& \cup \mid \\
& \mathcal{W}_{d}^{r}=\left\{L \rightarrow C \mid \operatorname{deg}_{C}(L)=d, g(C)=g, \text { and } h^{0}(C, L) \geqslant r+1\right\} .
\end{aligned}
$$

The fact that these are well-defined schemes with good properties is nontrivial, but is studied carefully in [ACGH].

The tangent space $T_{l}\left(\mathrm{Pic}^{d}\right)$ can be identified with $H^{1}\left(C, \Sigma_{L}\right)$, where $\Sigma_{L}$ is the extension of $\theta_{C}$ by $\Theta_{C}$,

$$
0 \rightarrow \theta_{C} \rightarrow \Sigma_{L} \rightarrow \Theta_{C} \rightarrow 0,
$$


with extension class

$$
\omega=c_{1}(L) \in H^{1}(C, \underline{\operatorname{Hom}}(\Theta, \theta)) \simeq H^{1}(C, K) .
$$

This leads (nontrivially) to the definition of $\mu_{1}: \operatorname{ker}\left(\mu_{0}\right) \rightarrow H^{0}\left(C, K^{2}\right)$ given by

$$
\mu_{1}\left(\sum_{i} s_{i} \otimes r_{i}\right)=\frac{\partial s_{i \alpha}}{\partial z_{\alpha}} \otimes r_{i \alpha} \in H^{0}\left(C, K^{2}\right),
$$

where $\sum_{i} s_{i} \otimes r_{i}$ is in $\operatorname{ker}\left(\mu_{0}\right)$.

Again, by studying obstructions, it can be seen that when $h^{0}(C, L)=r+1$, we have

$$
\operatorname{dim} T_{l}\left(\mho_{d, g}^{r}\right)=3 g-3+\rho+\operatorname{dim}\left(\operatorname{ker} \mu_{1}\right) .
$$

Thus in order to compute the dimension of $T_{l}\left(\mho_{6,10}^{2}\right)$, one must first find $\operatorname{ker}\left(\mu_{0}\right)$ and then the dimension of $\operatorname{ker}\left(\mu_{1}\right)$.

2. Begin by fixing $l=L \rightarrow C \in \mathcal{Q}_{6,10}^{2}-\mathcal{W}_{6,10}^{3}$, where $C$ is a smooth, genus 10 , hyper-elliptic curve. A simple investigation of the map associated to the linear system $|L|, \varphi_{|L|}: C \rightarrow \mathbf{P}^{2}$ (see [Griffin 2]) shows that, in this case, $|L|$ has two basepoints and is of the form $|L|=2 g_{2}^{1}+Q+R$, with $Q \neq i R$, where $i: C \rightarrow C$ is the involution on $C$. We may assume $|L|=|4 P|+Q+R$, where $P$ is a Weierstrass point on $C$.

In order to compute $\mu_{0}$, choose a basis for $H^{0}(C, L)=H^{0}(C, 4 P+Q+R)$, say $x_{0}, x_{1}, x_{2}$, where ord ${ }_{P}\left(x_{i}\right)=2 i$. Then a basis for

$$
H^{0}\left(C, K L^{-1}\right)=H^{0}\left(C, \Theta_{C}(10 P+i Q+i R)\right)
$$

is

$$
\left\{x_{0}^{2}, x_{0} x_{1}, x_{0} x_{2}, x_{1} x_{2}, x_{2}^{2}, y_{4}\right\} \quad \text { where ord } P\left(y_{4}\right)=10 \text {. }
$$

On the other hand, $H^{0}(C, K)=H^{0}\left(C, \Theta_{C}(18 P)\right)$ has basis $\left\{x_{0}^{3}, x_{0}^{2} x_{1}, x_{0}^{2} x_{2}, x_{0} x_{1} x_{2}, x_{0} x_{2}^{2}, x_{1} x_{2}^{2}, x_{2}^{3}, x_{2} y_{4}, z_{5}, z_{6}\right\} \quad$ where ord ${ }_{P}\left(z_{j}\right)=2 j+6$.

From this it is clear that $\mu_{0}$ is neither injective nor surjective ( $\mu_{0}$ just "multiplies" $\left.x_{i} \otimes x_{j} x_{k} \rightarrow x_{i} x_{j} x_{k}\right)$. Its kernel has dimension 10 and basis given in

\section{TABLE 1}

$$
\begin{aligned}
& v_{1}: x_{0} \otimes x_{0} x_{1}-x_{1} \otimes x_{0}^{2} \\
& v_{2}: x_{0} \otimes x_{0} x_{2}-x_{2} \otimes x_{0}^{2} \\
& v_{3}: x_{0} \otimes x_{1} x_{2}-x_{1} \otimes x_{0} x_{2} \\
& v_{4}: x_{0} \otimes x_{1} x_{2}-x_{2} \otimes x_{0} x_{1} \\
& v_{5}: x_{0} \otimes x_{2}^{2}-x_{2} \otimes x_{0} x_{2} \\
& v_{6}: x_{0} \otimes y_{4}-x_{2} \otimes x_{1} x_{2} \\
& v_{7}: x_{1} \otimes x_{1} x_{2}-x_{2} \otimes x_{0} x_{2} \\
& v_{8}: x_{1} \otimes x_{2}^{2}-x_{2} \otimes x_{1} x_{2} \\
& v_{9}: x_{1} \otimes y_{4}-x_{2} \otimes x_{2}^{2} \\
& v_{10}: x_{0} \otimes x_{0} x_{2}-x_{1} \otimes x_{0} x_{1}
\end{aligned}
$$


The elements $v_{6}, v_{7}, v_{9}, v_{10}$ come from relations in $H^{0}(C, K)$, namely $x_{0} x_{2}-x_{1}^{2}$ $=0, x_{0} y_{4}-x_{1} x_{2}^{2}=0$ and $x_{1} y_{4}-x_{2}^{2}=0$. The other elements are Koszul relations.

In order to compute $\operatorname{ker}\left(\mu_{1}\right)$ we will need

$$
\begin{aligned}
\frac{\partial x_{2 \alpha}}{\partial z_{\alpha}} & =\frac{\partial}{\partial z_{\alpha}}\left(\frac{x_{1 \alpha}^{2}}{x_{0 \alpha}}\right)=\frac{2 x_{1 \alpha} x_{0 \alpha}\left(\partial x_{1 \alpha} / \partial z_{\alpha}\right)-x_{1 \alpha}^{2}\left(\partial x_{0 \alpha} / \partial z_{\alpha}\right)}{x_{0 \alpha}^{2}} \\
& =\frac{2 x_{1 \alpha}\left(\partial x_{1 \alpha} / \partial z_{\alpha}\right)-x_{2 \alpha}\left(\partial x_{0 \alpha} / \partial z_{\alpha}\right)}{x_{0 \alpha}}
\end{aligned}
$$

which comes from the relation $x_{0} x_{2}-x_{1}^{2}$. The $\alpha$ subscript will be dropped (for obvious reasons) and the dependence will be denoted by \{\} 's.

We now give a table of $w_{i}=\mu_{1}\left(v_{i}\right)$.

TABLE 2

$$
\begin{aligned}
& w_{1}:\left\{x_{0} x_{1}\left(\partial x_{0} / \partial z\right)-x_{0}^{2}\left(\partial x_{1} / \partial z\right)\right\} \\
& w_{2}:\left\{2 x_{0} x_{2}\left(\partial x_{0} / \partial z\right)-2 x_{0} x_{1}\left(\partial x_{1} / \partial z\right)\right\} \\
& w_{3}:\left\{x_{1} x_{2}\left(\partial x_{0} / \partial z\right)-x_{0} x_{2}\left(\partial x_{1} / \partial z\right)\right\} \\
& w_{4}:\left\{2 x_{1} x_{2}\left(\partial x_{0} / \partial z\right)-2 x_{0} x_{2}\left(\partial x_{1} / \partial z\right)\right\} \\
& w_{5}:\left\{2 x_{2}^{2}\left(\partial x_{0} / \partial z\right)-2 x_{1} x_{2}\left(\partial x_{1} / \partial z\right)\right\} \\
& w_{6}:\left\{2 y_{4}\left(\partial x_{0} / \partial z\right)-2 x_{2}^{2}\left(\partial x_{1} / \partial z\right)\right\} \\
& w_{7}:\left\{x_{2}^{2}\left(\partial x_{0} / \partial z\right)-x_{1} x_{2}\left(\partial x_{1} / \partial z\right)\right\} \\
& w_{8}:\left\{y_{4}\left(\partial x_{0} / \partial z\right)-x_{2}^{2}\left(\partial x_{1} / \partial z\right)\right\} \\
& w_{9}:\left\{\left(x_{1} y_{4} / x_{0}\right)\left(\partial x_{0} / \partial z\right)-y_{4}\left(\partial x_{1} / \partial z\right)\right\} \\
& w_{10}:\left\{x_{0} x_{2}\left(\partial x_{0} / \partial z\right)-x_{0} x_{1}\left(\partial x_{1} / \partial z\right)\right\}
\end{aligned}
$$

Trivially, $w_{2}=2 w_{10}, w_{4}=2 w_{3}, w_{5}=2 w_{7}, w_{6}=2 w_{8}$, and in $H^{0}\left(C, K^{2} L\right)$ we have

$$
\begin{gathered}
x_{0} w_{2}=2 x_{1} w_{1}, \quad x_{1} w_{2}=2 x_{0} w_{3}, \quad x_{0} w_{5}=2 x_{1} w_{3}, \\
x_{0} w_{6}=x_{1} w_{5} \text { and } x_{1} w_{6}=2 x_{0} w_{9} .
\end{gathered}
$$

This clearly implies there can be no linear relation among $\left\{w_{1}, w_{2}, w_{3}, w_{5}, w_{6}, w_{9}\right\}$ in $H^{0}\left(C, K^{2}\right)$. Consequently, the kernel of $\mu_{1}$ has basis $\left\{w_{2}-2 w_{10}, w_{4}-2 w_{3}, w_{5}-\right.$ $\left.2 w_{7}, w_{6}-2 w_{8}\right\}$. By equation $(*)$ we finally have

$$
\begin{aligned}
\operatorname{dim} T_{l}\left(\mathcal{W}_{6,10}^{2}\right) & =3 g-3+\rho+\operatorname{dim}\left(\operatorname{ker} \mu_{1}\right) \\
& =3(10)-3+10-3(10-6+2)+4=23 .
\end{aligned}
$$

It is easy to compute the dimension of the hyper-elliptic component $W$ of $9 \int_{6,10}^{2}$. Since the $g_{2}^{1}$ on a hyper-elliptic curve is unique and $l=L \rightarrow C$, where $|L|=2 g_{2}^{1}+P$ $+Q$, one has immediately that

$$
\operatorname{dim} W=\operatorname{dim}\{\text { genus } 10 \text {, hyper-elliptic curves }\}+2 .
$$

By Hurwitz's formula,

$$
\operatorname{dim}\{\text { hyper-elliptic curves of genus } g\}=2 g-1 \text {. }
$$

Therefore,

$$
\operatorname{dim} W=19+2=21 \text { ! }
$$


Thus, since $\operatorname{dim} T_{l}\left(\mathcal{W}_{6,10}^{2}\right)=23>\operatorname{dim}{ }_{l}\left(\mathcal{W}_{6,10}^{2}\right)=21$, we conclude

THEOREM. The "component of hyper-elliptics" in o $_{6,10}^{2}$ is nonreduced.

A final remark: In general the singularities of $\mathcal{U}_{d, g}^{r}$ are "worst" along $\mathcal{Q} \int_{d, g}^{r+1}$, and in fact one can show, using these methods, that if $l \in \mathcal{Q} \int_{6,10}^{3}$,

$$
\operatorname{dim} T_{l}\left(\mho_{6,10}^{2}\right)>23 .
$$

In spite of this, $2 \int_{6,10}^{3}$ is smooth, i.e.

$$
\operatorname{dim} T_{l}\left(\text { W }_{6,10}^{3}\right)=\operatorname{dim}_{l}\left(\text { W }_{6,10}^{3}\right)=19
$$

for all $l \in \mathcal{W}_{6,10}^{3}$. So it is very important to distinguish between $T_{l}\left(\mathcal{W}_{d, g}^{r+1}\right)$ and $T_{l}\left(\mathcal{Q} \int_{d, g}^{r}\right)$ when $l \in \mathcal{W} \int_{d, g}^{r+1} \subseteq \mathcal{Q} \int_{d, g}^{r}$.

\section{REFERENCES}

[ACGH] E. Arbarello, M. Cornalba, P. A. Griffiths and J. Harris, Topics in the theory of algebraic curves, Princeton Univ. Press. (to appear).

[Griffin 1] E. Griffin, Special fibers in families of plane curves, Ph. D. Thesis, Harvard Univ., 1982.

[Griffin 2] _. The component structure of $0 \int_{6}^{2}$ (to appear).

Department of Mathematics, Harvard University, Cambridge, Massachusetts 02138

Current address: Department of Mathematics, University of California, Los Angeles, California 90024 